\title{
Concept learning with one relevant dimension in the squirrel monkey
}

\section{HERBERT WELLS AND KENNETH DEFFENBACHER}

UNIVERSITY OF WASHINGTON
Sixteen squirrel monkeys learned four discriminations in a procedure patterned on human concept learning (CL). Each discrimination involved one relevant and three irrelevant dimensions. A backward learning curve of pre-criterion trials was stationary, in agreement with some models of human $\mathrm{CL}$. But unexpectedly, a forward learning curve within the criterion run appeared to be incremental

Students of human concept learning (CL), e.g., Bourne (1966) have mentioned the value of a comparative psychology of CL. Studies of other primates would be of particular relevance. Previous studies of primate CL (e.g., Andrew \& Harlow, 1948) have used procedures such as sequences of 6-trial object discriminations. But in the typical human CL study, $S$ is given a different stimulus on each trial, and the whole array is specified by known relevant and irrelevant dimensions. The present study is an initial demonstration of CL in a convenient primate using procedures similar to those in human studies.

\section{Method}

Ss were 16 female squirrel monkeys maintained at $90 \%$ ad lib weight.

The apparatus was a manually-operated WGTA with two Kluver-type pull-in carts replacing the single tray. In action, the carts were placed $7^{\circ}$ in. apart, 14 in. from the animals, and the cord from each cart was tied to a ring just outside the bars of S's compartment. A movable stimulus block covered the reward cup on each cart.

Stimuli were mounted on the face of a $2 \times 2$ in. wood block, and represented all 81 combinations of size (large, medium, small); shape (triangle, circle, square); color of background (green, yellow, blue); and pattern within the figure (a cross, vertical stripes, crosshatching).

In each discrimination problem, Ss were presented with a choice between two stimuli on each of 36 trials per day, in a non-correction procedure. Positive and negative stimuli were randomly paired, and correct side was randomized with a restriction of three consecutive placements on the right or left cart. A criterion of 15 out of 18 consecutive correct responses was used.

Ss learned four discriminations in a series of nonreversal shifts. In each case there was one relevant and three irrelevant dimensions. The first problem also served as a shaping task, in which the choices were made successively more complex by the addition of irrelevant dimensions. The positive cues in the four problems were Small, Circle, Stripes, and Green. A Latin square ensured that problems were encountered in different orders.

\section{Results and Discussion}

The four successive problems were learned in an average of $75.6,146.9,143.2$, and 131.7 trials to criterion. The first problem appears to be learned more rapidly only because data were not recorded until shaping had been completed. There was no reliable improvement over the four problems. Nor were there reliable differences among the four relevant dimensions. Mean trials to criterion were: Small= 135.6; Circle=129.6; Stripes $=142.5 ;$ Green $=89.7$. In a specific comparison, Green did not differ from the other dimensions. These general results for the Latin square analysis of variance were not unexpected, since the stimulus dimensions had been chosen to be relatively comparable, and since this species is not noted for rapid learning-to-learn (Harlow, 1959; Miles, 1957).

How do these data compare with those from human studies using similar procedures? Some recent models of human CL (e.g., Bower \& Trabasso, 1964) predict one-trial learning of simple, one-relevant-dimension concepts of the kind used here. The principal indication of this is a flat, "stationary" backward learning curve prior to the last error before criterion. Such a curve appears in the left-hand portion of Fig. 1, based on data from the last three problems for all $16 \mathrm{Ss}$, potentially 48 scores per trial. (The shaping involved in the first problem makes it non-comparable.) A non-stationary analysis (Suppes \& Ginsberg, 1963) yields a Chi square of only $5.36(d f=9 ; p>.80)$, indicating that the curve is indeed stationary rather than incremental. Subject loss is modest: there are 44 scores per trial in Block -1, 43 in $-4,41$ in -8 , and 35 in -10 .

The right-hand portion of the graph shows blocks of four trials within the criterion, plus the final two

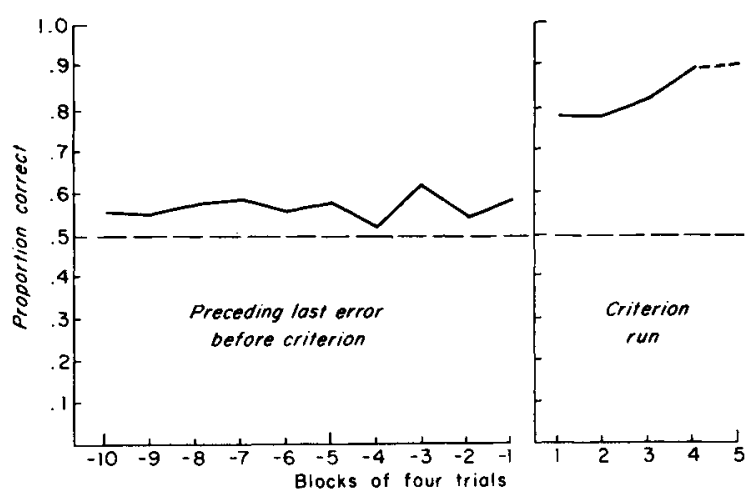

Fig. 1. Proportion of correct responses in blocks of four trials. 
trials. In all cases there are 48 scores per trial. The increase in the first four blocks is significant $\left(x^{2}=\right.$ $11.29, \mathrm{df}=3, \mathrm{p}<.02$ ). This unexpected result resembles data presented by Suppes \& Ginsberg (1963) for the final portions of Vincentized backward learning curves when criteria of perfect performance are imposed on human Ss. These authors, as well as Restle (1964), propose a model which predicts a multi-stage process when more than one cue must be conditioned. In these problems, though, a single cue would suffice. One interpretation is that following the initial rapid improvement (adoption of the correct "hypothesis"?) Ss garner a higher proportion of reinforcement which allows a rapid incremental phase (confirmation?).

However, the present data have been examined not as a typical backward learning curve, but as an "inside out" curve with both backward and forward components. While this was necessary because of the less-thanperfect criterion, it has unique statistical properties. In particular, the criterion mun portion, as any forward learning curve, may appear incremental by averaging individual step functions which occur on different trials. Further comparative work will explore some of these problems, and will also apply these techniques to more complex concept tasks.

\section{References}

Andrew, G., \& Harlow, H. F. Performance of macaque monkeys on a test of the concept of generalized triangularity. Comp. Psychol. Monogr., 1948, 19, No. 3 (Serial No. 100).

Bourne, L. E. Human conceptual behavior. Boston: Allyn and Bacon, 1966.

Bower, G. H., \& Trabasso, T. R. Concept identification. In R. C. Atkinson (Ed.), Studies in mathematical psychology. Stanford: University Press, 1964.

Harlow, H. F. Learning set and error factor theory. In S. Koch (Ed.), Psychology, a study of a science, Vol. 2. New York: McGrawHill, 1959.

Miles, R.C. Learning set formation in the squirrel monkey. $J$. comp. physiol. Psychol., 1957, 50, 356-357.

Restle, F. Sources of difficulty in learning paired associates. In R. C. Atkinson (Ed.), Studies in mathematical psychology. Stanford: Stanford University Press, 1964.

Suppes, P., \& Ginsberg, Rose. A fundamental property of all-ornone models.... Psychol. Rev., 1963, 70, 139-161.

\section{Note}

1. This research was supported by the Research Fund of the Graduate School, University of Washington, and by Public Health Service Research Grant MH 12309-01. 